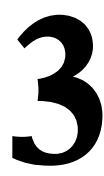

\title{
DISEÑO Y VALIDACIÓN DE UN INSTRUMENTO DE MEDIDA DEL CLIMA EN CENTROS DE EDUCACIÓN SECUNDARIA
}

\section{(DESIGN AND VALIDATION OF A CLIMATE MEASURMENT INSTRUMENT IN SECONDARY SCHOOLS)}

Jesús Miguel Rodríguez Mantilla

Universidad Camilo José Cela

M. José Fernández Díaz

Universidad Complutense de Madrid

DOI: $10.5944 / e d u c X X 1.18 .1 .12312$

\section{Cómo referenciar este artículo/How to reference this article:}

Rodríguez Mantilla, J. M. y Fernández Díaz, M. J. (2015). Diseño y validación de un instrumento de medida del clima en centros de educación secundaria. Educación XX1, 18(1), 71-98. doi: 10.5944/educXX1.18.1.12312

Rodríguez Mantilla, J. M. y Fernández Díaz, M. J. (2015). Design and validation of a climate measurement instrument in Secondary Schools. Educación XX1, 18(1), 71-98. doi: 10.5944/ educXX1.18.1.12312

\section{RESUMEN}

El presente estudio forma parte de una investigación acerca de la influencia del clima institucional en el síndrome de burnout. El objetivo, en este caso, es elaborar y validar un instrumento de medida del clima de los centros de Secundaria. Tras la elaboración y aplicación del instrumento a una muestra de 794 docentes de la Comunidad de Madrid, se analizó la fiabilidad, la validez de contenido y de constructo (esta última a través de Modelos de Ecuaciones Estructurales con la aplicación informática AMOS 7.0). Los resultados obtenidos en el análisis de fiabilidad (alfa de Cronbach $=0,947$ para la totalidad del instrumento, 0,842, 0,923 y 0,964 para las dimensiones de Relación Profesor-Alumnos, Profesor-Compañeros y Profesor-Superiores respectivamente) y en el Análisis Factorial Confirmatorio fueron altamente satisfactorios $(\mathrm{CMIN} / \mathrm{DF}=2,899, \mathrm{CFI}=0,904, \mathrm{RMSEA}=0,049, \mathrm{PRATIO}=0,946)$. Los valores del coeficiente de discriminación de los ítems del instrumento final oscilaron entre 0,29 y 0,78 . Por todo ello, el instrumento presentado reúne las características técnicas exigidas para ser considerada una herramienta válida y fiable para medir el clima de los centros. 


\title{
PALABRAS CLAVE
}

Clima, profesorado, educación secundaria.

\begin{abstract}
This study is part of research on the influence of institutional climate on burnout syndrome. The aim in this case is to develop and validate an instrument to measure school climate in Secondary Education. After the development and application of the instrument to a sample of 794 teachers in the Community of Madrid, reliability, content and construct validity were analyzed (the latter using Structural Equations Models with the software AMOS 7.0). The results obtained in the analysis of reliability (Cronbach's alpha $=0.947$ for the totality of the instrument, $0.842,0.923$ and 0.964 for the dimensions of Teacher-Student, Teacher-College and Teacher-Superior Relationships respectively) and in the Confirmatory Factorial Analysis were highly satisfactory $(\mathrm{CMIN} / \mathrm{DF}=2.899, \mathrm{CFI}=0.904, \mathrm{RMSEA}=0.049$, PRATIO = 0.946). The discrimination coefficient values of the final instrument items ranged between 0.29 and 0.78 . Therefore, this instrument has proved to have the necessary technical characteristics to be considered a valid and trustworthy tool to measure the syndrome.
\end{abstract}

\section{KEY WORDS}

Organizacional climate, teacher, secondary education.

\section{INTRODUCCIÓN}

La realidad actual, en el ámbito educativo, muestra un perfil muy diferente al de hace años, debido, en gran parte, a los vertiginosos cambios sociales que han influido en el campo de la educación. Cierto es que existen novedades en el campo educativo que abren nuevas vías de investigación - como es el caso de la inclusión de las nuevas tecnologías en las aulas (Rebollo, García, Barragán, Buzón y Ruiz, 2012)—, pero otros factores siguen siendo objeto de estudio desde hace décadas, si bien su perspectiva de estudio ha ido evolucionando con los años. Este es el caso del clima social en los centros educativos (Murillo y Becerra, 2009).

El clima de una institución se puede ver afectado por variables de muy diversa naturaleza, en particular de tipo estructural, tales como el estilo de dirección, políticas y planes de gestión, el tamaño de la organización, etc., pudiendo afectar a la posible aparición de satisfacción profesional (Informe Cisneros XI, 2009; Murillo y Becerra, 2009; Rivas, Leite y Cortés, 2011) o al desarrollo de síndromes como el burnout (en el caso de encontrar una ex- 
cesiva presión, conflictos y un bajo nivel de recompensas, reconocimientos y éxitos laborales. Rodríguez Mantilla y Fernández Díaz, 2012). Queda, por tanto, de manifiesto que el clima de las organizaciones guarda una estrecha relación con las interacciones sociales de sus miembros.

Diversos estudios señalan la importancia del papel que juega el clima de las instituciones sobre los modos de hacer, sentir y pensar de los miembros de una organización y, por tanto, en el modo en que esta vive y se desarrolla, (Informe Cisneros XI, 2009; Wilkins y Kuperminc, 2010). Entendiendo el clima laboral u organizacional como la percepción que tienen los sujetos acerca de las relaciones interpersonales que se establecen en el contexto de trabajo y el marco en el que estas interacciones se dan (Guerra et al., 2012), cabe señalar que fue la corriente cognitivista la primera en mostrar su interés por el estudio en este campo, señalando la importancia que la percepción tiene a la hora de influir en la realidad misma. Es decir, cada persona percibe de modo distinto el contexto en el que se desenvuelve y dicha percepción influye en el comportamiento del individuo en la organización, con todas las implicaciones que ello conlleva. La percepción que los sujetos hacen de la situación está influenciada por cuestiones internas y externas a ellos (aspectos psíquicos, anímicos, familiares, sociales, de educación y económicos que rodean la vida de cada individuo), condicionando su visión del clima (Cano-García, Padilla-Muñoz y Carrasco-Ortiz, 2005).

Concretamente, en el contexto escolar, Molina y Pérez (2006) señalan que los centros educativos son, ante todo, organizaciones sociales, ya que están conformadas por un grupo de personas que ejercen determinadas funciones ordenadas por fines, objetivos y propósitos, dirigidos hacia la búsqueda de la eficiencia y la racionalidad, siendo la realidad fenomenológica de dicha organización lo que conforma el clima. Texeidó Saballs y Capell Castañar (2002) señalan que las relaciones humanas juegan un papel muy importante en las instituciones escolares, puesto que durante la actividad educativa se produce un proceso recíproco mediante el cual las personas que se ponen en contacto, valoran los comportamientos de los otros y se forman opiniones acerca de ellos, lo que suscita sentimientos que influyen en el tipo de relaciones que se establecen. Todo centro educativo, como organización social, debe buscar un sistema de comunicación e información que dinamice los procesos. Las comunicaciones internas en una organización promueven la participación, la integración y la convivencia en el marco del clima, donde cobra sentido el ejercicio de funciones y el reconocimiento de las capacidades individuales y grupales.

Como también señalan Ruz-Primo, Jornet y Backhoff (2006), son muchos los estudios que se centran en las características de los centros educativos (a nivel de organización y de aula) y su relación con los resultados (en 
términos de logros de aprendizaje, bienestar personal entre sus miembros, eficacia en la gestión, etc.). De ahí que el estudio del clima se haya convertido en una de las áreas de investigación educativa de mayor relieve en el ámbito internacional. Estudios realizados han demostrado que el clima en el que trabajan las personas es fundamental para el éxito, el desarrollo de buenos resultados productivos de una organización y la satisfacción laboral. Como consecuencia de esa satisfacción laboral, Arciniega (2002) señala que un profesor puede desarrollar un mayor compromiso con el centro de trabajo, en cuyo caso su nivel de productividad será mayor y su índice de absentismo más bajo. En este sentido, Hernández y Olmedo (2004) y CanoGarcía et al. (2005) afirman que el clima influye en la satisfacción docente, en la productividad, en el grado de compromiso y en el comportamiento del individuo.

La existencia de múltiples protagonistas en una institución escolar (profesores, alumnos, apoderados, auxiliares, inspectores, directivos, etc.) hace que el clima pueda ser abordado desde distintos enfoques y que podamos encontrar una gran variedad de opiniones, percepciones y, como consecuencia de ello, diversidad en los instrumentos de medida. En este caso, nos centraremos en dos tipos de cuestionarios: los dirigidos a alumnos y los dirigidos a profesores. Entre los primeros, encontramos instrumentos diseñados para evaluar el clima relacional de los alumnos con sus iguales y profesores, tales como el School Climate Survey Form (Kelley, 1989), el School Climate Scale (Haynes, Emmons y Ben-Avie, 1997), el California School Climate and Safety Survey (Rosenblatt y Furlong, 1997), la escala de Conducta y Experiencias Sociales en Clase (Collell y Escudé, 2006), el Cuestionario para evaluar el Clima Social del centro escolar (Trianes et al., 2006) o la Escala para la valoración de la variable Clima Social de Aula en alumnos de Educación Primaria y Secundaria (Pérez, Ramos y Lopez, 2009). Por otro lado, entre los instrumentos de medida dirigidos a evaluar el clima de centro percibido por los profesores podemos encontrar la Escala de Evaluación del Clima de Centros Educativos (Fernández y Asensio, 1993), el Inventario sobre la Salud de la Organización (Hoy y Feldman, 1999), la Escala Sistémica de Observación de la Clase (Baeza, 2005), la Escala de Diagnóstico Organizacional (Rodríguez, 2005) o el Instrumento de Medida del Clima Organizacional en Educación Superior (Mejías, Reyes y Arzola, 2006).

La literatura especializada deja patente el papel determinante que tienen las relaciones interpersonales en la configuración del clima social de los centros. Esparza, Guerra y Martínez (2000) señalan que, dentro de una institución, podemos distinguir microclimas que pueden relacionarse entre sí, como son: el clima de los alumnos, el clima entre alumnos y profesores, el clima entre profesores, el clima entre superiores y pro- 
fesorado, etc. Si estas relaciones que se establecen son negativas, señalan los autores, se puede configurar un factor de riesgo en la manifestación de síndromes como depresión o burnout. Este estudio se centrará en tres de estas relaciones: Relación Profesor-Alumno, Relación Profesor-Compañeros y Relación Profesor-Superiores.

En las últimas décadas, la Relación Profesor-Alumno ha ido mostrando, especialmente en el caso de la Educación Secundaria Obligatoria (ESO), una problemática cada vez más compleja. Esteve (2009) y el Informe Cisneros XI (2009) señalan que la escolaridad obligatoria hasta los 16 años parece conllevar, en ocasiones, la incorporación al sistema educativo de alumnos que manifiestan abiertamente su desinterés por el estudio a través de conductas disruptivas en las aulas y de un rechazo hacia las normas escolares. En este sentido, un clima negativo dentro del aula se asocia a efectos negativos sobre el ajuste psicológico de los sujetos (Kuperminc, Leadbeater y Blatt, 2001; Westling, 2002) y a un aumento del riesgo de conductas agresivas y violentas que pueden impactar negativamente en la vida del aula o del centro. Concretamente, autores como Cano-García et al. (2005) señalan la importancia de la Relación Profesor-Alumno como factor determinante para disminuir el absentismo de los docentes, el riesgo de aparición de depresión, burnout, etc. Jennings y Greenberg (2009) señalan que, entre las características que deben configurar un clima óptimo, se han de encontrar niveles bajos de conflicto y de comportamiento quebrantador, orden y organización de la clase, comunicación respetuosa, de apoyo y sensibilidad a las diferencias individuales y las necesidades de los estudiantes. Esta definición deja patente el papel que juegan las habilidades del profesor para mantener un clima y trato adecuado con los alumnos, y a este respecto Marzano, Marzano y Pickering (2003), señalan que cuando los profesores carecen de recursos para manejar de forma eficaz los desafíos sociales y emocionales dentro del contexto de la clase, el clima de aula se deteriora, aumentando así los conflictos con los alumnos y la pérdida de control a la hora de manejar la situación. Jennings y Greenberg (2009) destacan la importancia de la capacidad social y emocional de los profesores en el desarrollo y mantenimiento de las relaciones entre profesor-alumno, señalando que los profesores competentes a nivel socio-emocional son aquellos que reconocen sus emociones, fuerzas y debilidades, generan emociones, motivan, tienen alta conciencia social, son empáticos, ponen límites, etc.

En el campo de la Relación Profesor-Compañeros, un factor determinante es la aparición de conflictos entre profesores (por roles mal entendidos, falta de responsabilidad de algunos compañeros, problemas de comunicación, incumplimiento de normas, etc.). No debemos olvidar que los centros son organismos vivos y la realidad es que la mayoría presenta situaciones conflictivas debidas, en ocasiones, a la deficiente comunica- 
ción de los individuos y la interferencia de las actitudes personales en la relación laboral. Sin embargo, hay que entender que el conflicto no tiene por qué ser algo únicamente negativo. El conflicto es una instancia de aprendizaje dentro del proceso de crecimiento de cualquier grupo social, por lo que hay que admitirlos como parte del desarrollo institucional (Ruz-Primo, Jornet y Backhoff, 2006). Por su parte, Esparza et al. (2000), Aciego, Domínguez y Hernández (2003) y Romasz, Cantor y Elias (2004) señalaron la importancia de la Relación Profesor-Compañeros en la adaptación laboral, la capacidad de afrontamiento, autoconcepto, empatía y sociabilidad y su implicación en la aparición de síndromes depresivos y de burnout en los profesores.

En el ámbito de la Relación Profesor-Superiores, autores como Lozano (2013), encontraron una relación positiva significativa entre liderazgo transformacional y la motivación de los empleados, y el grado de compromiso con la calidad de las consecuencias organizacionales. Gillespie y Mann, (2004), por su parte, encontraron que el rol del liderazgo transformacional tiene un papel en la predicción de la confianza de los subordinados, lo que influye en la efectividad del líder.

Ante esta realidad, el presente trabajo tiene como principal objetivo diseñar y desarrollar un instrumento de medida válido y fiable, fundamentado en una definición conceptual y operativa, que reúna las características psicométricas exigidas para medir el clima social de los centros en el ámbito español. Para la elaboración del instrumento se partió de la configuración de tres dimensiones: Relación Profesor-Alumno, Relación Profesor-Compañeros y Relación Profesor-Superiores.

\section{MÉTODO}

\section{Muestra}

La población de estudio corresponde a los docentes de ESO de la Comunidad Autónoma de Madrid (CAM), formada por un total de 13.318 docentes (Consejería de Educación de Madrid, 20013). Para ello se accedió a un total de 1.291 profesores de ESO correspondientes a un total de 38 centros de las distintas zonas (norte, sur, este, oeste y centro) de la CAM, de los que, finalmente, 794 participaron en el estudio, obteniendo una tasa de respuesta del 61,50\%. Hair, Anderson, Tathan y Black (2009) señalan que, como regla general, es conveniente contar, como mínimo, con un número de observaciones cinco veces mayor que el número de variables, siendo, no obstante, el tamaño aceptable de un ratio de diez a uno. Nuestra muestra consta de 794 observaciones y el instrumento de medida, como 
veremos más adelante, está compuesto por 60 ítems, por lo que obtenemos un ratio de observaciones/variables de 13,23, situado por encima de lo recomendado.

Los profesores fueron seleccionados a través de un muestreo incidental, de tal manera que el $62,6 \%$ de la muestra $(n=497)$ pertenece a centros públicos, el $29,85 \%(n=237)$ a centros concertados y el $7,55 \%(n=60)$ a centros privados. Esta distribución se ajusta, tanto por tipo de centro como por zonas, a la distribución de la población de la CAM (Consejería de Educación de Madrid, 2009). La distribución por zonas del profesorado refleja que el $14,1 \%(\mathrm{n}=112)$ pertenecen a la zona norte de la Comunidad, el $34,4 \%$ $(n=273)$ a la zona sur, el $12,6 \%(n=100)$ a la zona este, el $14,9 \%(n=118)$ a la zona oeste y el $24,1 \%(n=191)$ a la zona centro.

En cuanto a las características sociodemográficas, la muestra está formada por 318 mujeres $(40,1 \%)$ y 476 varones $(59,9 \%)$. El rango de edad de los profesores menores de 39 años corresponde al 45,2\% de la muestra, mientras que el $34,6 \%$ de los sujetos tiene entre 40 y 49 años $(n=275)$. De este modo, un $20,2 \%$ de los docentes tiene 50 años o más.

Por último, desde el punto de vista del perfil profesional, el 20,2\% de la muestra tiene 4 años o menos de experiencia docente, el $27,1 \%$ entre 5 y 10 años, el 22,5\% entre 11 y 19 años. Así pues, el 30,2 \% restante posee 20 años o más de experiencia como profesor.

\section{Instrumento}

La medición del clima social de los centros se llevó a cabo a través de un instrumento elaborado expresamente para la ocasión, formado por ítems que refieren a la relación interpersonal del profesor con compañeros, superiores y alumnos. De esta manera, el cuestionario quedó configurado por un total de 60 ítems (ver Tabla 1) a los que el profesor debía responder atendiendo a una escala de 1 a 5 de tipo Likert (donde 1 indica Nada, nunca, y 5 indica Mucho, siempre), para todos los ítems de las distintas dimensiones.

\section{Procedimiento}

Para conseguir la participación de los centros y de los profesores en el estudio, el equipo de investigación proporcionó los cuestionarios a los centros, acompañados de una carta informativa sobre el objeto de estudio, informando y asegurando el anonimato de los participantes. Los cuestionarios fueron depositados, una vez cumplimentados por los profesores, en un 
buzón habilitado en los centros para dicho fin. De igual modo, se aseguró a los equipos directivos la remisión de la información y resultados obtenidos en los análisis, con el objeto de que la colaboración de los centros fuese de utilidad para ellos mismos a nivel interno.

\section{Elaboración del cuestionario}

Para la elaboración de la escala de medida del clima se diseñó un sistema de dimensiones, subdimensiones e indicadores sustentado en las aportaciones de autores tan relevantes como Kuperminc et al. (2001), Westling (2002), Esteve (2009), Cano-García et al. (2005), Collell y Escudé (2006) y Jennings y Greenberg (2009), entre otros, adaptando sus teorías al contexto educativo de nuestro país y al de la etapa de Secundaria. De este modo, el instrumento quedó configurado por tres grandes dimensiones: Relación Profesor-Alumno, Relación Profesor-Compañeros y Relación Profesor-Superiores (Tabla 1).

La Relación Profesor-Alumno quedó compuesta de las subdimensiones comportamiento de los alumnos (modo en que los estudiantes se dirigen al profesor, grado en que cumplen las normas de disciplina y existencia de agresiones por parte de los alumnos a los profesores, a nivel físico o psicológico), interacción profesor-alumno (tipo de comunicación que mantiene el profesor con los alumnos y figura de apoyo y confianza de éste para los estudiantes ante problemas) y motivación del alumnado frente al desarrollo de las clases. En la dimensión Relación Profesor-Compañeros se incluyeron las subdimensiones de conflictos entre profesores (existencia de conflictos de tipo personal y profesional entre los docentes, presión entre profesores y nivel de compañerismo), interacción profesor-compañeros (tipo de relación que mantienen entre sí y sentimiento de valoración por parte de los demás), percepción de la profesionalidad de los compañeros (trabajo en equipo entre los profesores, cumplimiento de horarios, mantenimiento de la disciplina de los alumnos, competencia profesional, implicación y compromiso de los docentes con el centro y la actitud de los mismos) y trabajo en equipo (forma de trabajo entre profesores e implicación de los docentes en actividades comunes). Por último, la dimensión Relación Profesor-Superiores quedó configurada por las subdimensiones de liderazgo (actitud de los líderes, capacidad para resolver de forma eficaz los conflictos, problemas y necesidades de los profesores, capacidad de organización del trabajo, comunicación y actitud con el profesorado), en qué medida y cómo los líderes son fuente de motivación para el profesorado (reconocimientos y recompensas que los líderes otorgan a los profesores, participación del profesorado en la toma de decisiones en cuestiones relevantes para ellos y preocupación de los superiores por la formación y 
actualización profesional de sus profesores) y valoración profesional de los superiores (grado de profesionalidad de los superiores percibido por los profesores).

Posteriormente, para obtener evidencias que garantizasen la validez de contenido del instrumento, fundamentada por la definición de estas dimensiones, se seleccionaron, por un lado, expertos en investigación educativa con amplios conocimientos en la materia y en la elaboración y análisis de escalas, con el fin de juzgar de manera independiente los ítems del instrumento; y, por otro, profesionales del ámbito de ESO, con objeto de conocer la opinión de algunos profesores de la etapa respecto a los ítems incluidos en el instrumento. El grupo de jueces quedó finalmente compuesto por tres expertos y tres profesores de ESO. Para poder desempeñar correctamente las funciones de jueces críticos, cada uno de ellos fue informado acerca del propósito de la prueba y de la conceptualización del universo de contenido. Cada juez recibió un instrumento de validación en el que se recogió la información aportada por ellos. El experto debía valorar en una escala de 1 a 5 la relevancia (nivel de significatividad o importancia del ítem respecto a la dimensión en la que se encuadra) y la claridad de cada ítem del cuestionario. Por último, el instrumento de validación incluía una serie de preguntas abiertas sobre la pertinencia de añadir, eliminar o modificar alguno de los ítems presentados. Una vez evaluadas las escalas por el grupo de jueces, se procedió al análisis de los resultados, teniendo en cuenta ciertos aspectos básicos (Tejero, 2006 y Tejero, Fernández y Carballo, 2010): a) los ítems donde hubiera un 100\% de coincidencia favorable entre los jueces quedarían incluidos en el instrumento, b) los ítems donde hubiera un 100\% de coincidencia desfavorable entre los jueces, serían excluidos del instrumento y c) los ítems donde sólo hubiese coincidencia parcial entre los jueces deberían ser revisados.

A nivel cuantitativo, y siguiendo a Cortada de Kohan (1999), se valoró la posible eliminación de aquellos ítems que no superasen una media de 4 tanto en claridad como en relevancia, y aquellos que tuvieran una desviación típica mayor de 1,5. Sin embargo, y junto a ello, se tuvo en cuenta un análisis cualitativo y detallado de las respuestas a las preguntas abiertas formuladas en el instrumento de validación de todos y cada uno de los ítems. Las valoraciones de los expertos mostraron la alta relevancia de la totalidad de los ítems propuestos para el instrumento, por lo que los cambios y correcciones que se hicieron fueron leves correcciones ortográficas y gramaticales y algunas modificaciones en la redacción de algunos ítems para mejorar la claridad y la representatividad del indicador al que pertenecen. De este modo, los ítems incluidos en el instrumento se muestran en la Tabla 1. 


\begin{tabular}{|c|c|c|}
\hline DIM & INDICADORES & ITEMS \\
\hline \multirow{3}{*}{ 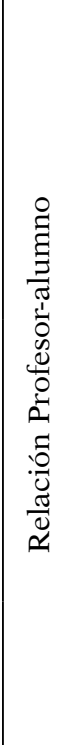 } & $\begin{array}{l}\text { Comportamiento } \\
\text { alumnos }\end{array}$ & $\begin{aligned} \text { 6. Durante mis clases los alumnos utilizan «palabrotas» o malas } \\
\text { formas en el aula. } \\
\text { 9. Los alumnos utilizan malas maneras cuando se dirigen a mí. } \\
\text { 13. En mi clase se respetan las normas de comportamiento. } \\
\text { 1. En general, en mis clases, los alumnos permiten que se imparta } \\
\text { la clase. } \\
\text { 11. Los alumnos respetan la autoridad de los profesores. } \\
\text { 12. En los últimos años he sufrido agresiones (verbales, físicas, inti- } \\
\text { midaciones o amenazas, etc.) por parte de algún/os alumno/os. }\end{aligned}$ \\
\hline & $\begin{array}{l}\text { Interacción } \\
\text { profesor-alumno }\end{array}$ & $\begin{array}{l}\text { 5. Los alumnos acuden a mí cuando tienen dificultades académi- } \\
\text { cas y no solo por cuestiones de examen. } \\
\text { 7. Además de los temas académicos, en la clase solemos tratar } \\
\text { otros aspectos personales y de la vida diaria. } \\
\text { 10. Los alumnos me confían sus temas o problemas personales. } \\
\text { 15. Cuando existen conflictos entre mis alumnos, acuden a mí. } \\
\text { 4. Existe una buena comunicación entre mis alumnos y yo. } \\
\text { 14. Mi relación con los alumnos es cercana. }\end{array}$ \\
\hline & $\begin{array}{l}\text { Motivación } \\
\text { alumnos }\end{array}$ & $\begin{array}{l}\text { 3. En mis clases los alumnos participan activamente. } \\
\text { 8. En general, noto que los alumnos sienten desmotivación hacia } \\
\text { mi asignatura. } \\
\text { 2. En general, la actitud de los alumnos es pasiva. }\end{array}$ \\
\hline \multirow{3}{*}{ 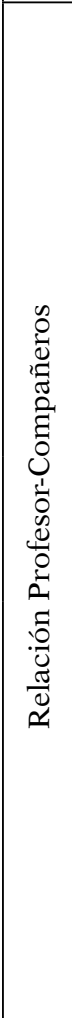 } & \multirow[t]{2}{*}{$\begin{array}{l}\text { Trabajo en } \\
\text { equipo }\end{array}$} & $\begin{array}{l}\text { 41. En general, los profesores comparten materiales y recursos con } \\
\text { otros profesores. } \\
\text { 29. En general, los profesores prestan su ayuda a un compañero } \\
\text { cuando tiene algún problema o dificultad. } \\
\text { 21. En general, los profesores son individualistas y van cada uno } \\
\text { a lo suyo. } \\
\text { 20. El profesorado se implica en los problemas del Centro. } \\
\text { 40. Considero que el profesorado se siente orgulloso de su Centro. } \\
\text { 25. A la hora de preparar ciertos eventos (Navidad, semana de la } \\
\text { ciencia, etc.) los profesores colaboran. } \\
\text { 36. Me siento satisfecho con el trabajo que desarrollan los profe- } \\
\text { sores en general. } \\
\text { 32. El profesorado manifiesta optimismo, energía y entusiasmo. }\end{array}$ \\
\hline & & $\begin{array}{l}\text { 16. Existe una implicación y actitud positiva por parte de los pro- } \\
\text { fesores a la hora de trabajar en equipo. } \\
\text { 22. En general, la comunicación entre los profesores de mi centro } \\
\text { es buena. }\end{array}$ \\
\hline & $\begin{array}{l}\text { Profesionalidad } \\
\text { de los } \\
\text { compañeros }\end{array}$ & $\begin{array}{l}\text { 17. En general, los profesores saben mantener el orden en sus } \\
\text { clases. } \\
\text { 24. Algunos de mis compañeros deberían manejar mejor el control } \\
\text { disciplinario de sus clases. } \\
\text { 30. Siento que mantengo la disciplina de mis alumnos de forma } \\
\text { más adecuada que la mayoría de mis compañeros. } \\
\text { 33. En general, considero que la falta de asistencia a clase de los } \\
\text { profesores es alta. } \\
\text { 37. En general, los profesores cumplen con los horarios de vigilan- } \\
\text { cias (por ejemplo: guardias de recreo, vigilancia de pasillos, etc.). } \\
\text { 39. lonsidero que el profesorado actúa en su trabajo con profe- } \\
\text { sionalidad. }\end{array}$ \\
\hline
\end{tabular}


DISEÑO Y VALIDACIÓN DE UN INSTRUMENTO DE MEDIDA DEL CLIMA EN CENTROS DE EDUCACIÓN...

\begin{tabular}{|c|c|c|}
\hline DIM & INDICADORES & ÍTEMS \\
\hline \multirow{2}{*}{ 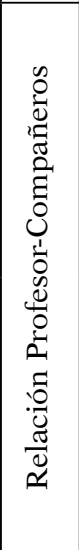 } & $\begin{array}{l}\text { Conflictos entre } \\
\text { profesores }\end{array}$ & $\begin{array}{l}\text { 35. He tenido o tengo conflictos de tipo profesional con otros pro- } \\
\text { fesores. } \\
\text { 23. He tenido o tengo conflictos de tipo personal con otros profesores. } \\
\text { 31. Me siento criticado negativamente por otros profesores. } \\
\text { 28. Siento que la actitud de algunos compañeros me dificulta ex- } \\
\text { presar mis opiniones. } \\
\text { 26. Hay un ambiente de continua disputa y competencia entre el } \\
\text { profesorado. }\end{array}$ \\
\hline & $\begin{array}{l}\text { Interacción entre } \\
\text { compañeros }\end{array}$ & $\begin{array}{l}\text { 38. En general, creo que la opinión que tienen mis compañeros de } \\
\text { mí es buena. } \\
\text { 18. Me siento valorado por mis compañeros. } \\
\text { 34. Las propuestas que hago son tenidas en cuenta por mis com- } \\
\text { pañeros. } \\
\text { 27. Mantengo buenas relaciones con todos mis compañeros. } \\
\text { 19. A nivel general, todos los profesores tenemos buenas relaciones } \\
\text { entre nosotros. }\end{array}$ \\
\hline \multirow{4}{*}{ 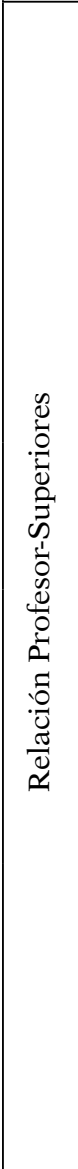 } & \multirow[t]{2}{*}{ Liderazgo } & $\begin{array}{l}\text { 57. Existe una buena y fluida comunicación entre el profesorado y } \\
\text { los cargos de responsabilidad (jefe de estudio, dirección, etc.). } \\
\text { 59. El jefe de estudios y yo mantenemos una buena comunicación } \\
\text { sobre cuestiones profesionales. } \\
\text { 45. El director y yo mantenemos una buena comunicación. } \\
\text { 43. Ante un problema profesional de algún profesor, la jefatura de } \\
\text { estudios (o dirección) responde de forma eficaz. } \\
\text { 44. El Equipo Directivo se muestra flexible a la hora de tener que } \\
\text { modificar sus decisiones. } \\
\text { 60. Los profesores confiamos profesionalmente en el director. } \\
\text { 49. La línea de actuación del equipo directivo se fundamenta más } \\
\text { en la improvisación que en la previsión o claridad de ideas. } \\
\text { 58. El director mantiene un trato adecuado conmigo. }\end{array}$ \\
\hline & & $\begin{array}{l}\text { 56. El Equipo Directivo mantiene buenas relaciones con el profe- } \\
\text { sorado en general. } \\
\text { 52. El jefe de estudios mantiene un trato adecuado con los profe- } \\
\text { sores. }\end{array}$ \\
\hline & $\begin{array}{l}\text { Valoración de los } \\
\text { superiores }\end{array}$ & $\begin{array}{ll}\text { 54. Me siento satisfecho con el trabajo que realiza el director. } \\
\text { 42. La jefatura de estudios atiende las necesidades (materiales, de } \\
\text { información, etc.) de los profesores. } \\
\text { 47. Considero que la jefatura de estudios hace un buen trabajo. } \\
\text { 48. La gestión y organización de eventos, tareas, etc. por parte de } \\
\begin{array}{l}\text { la jefatura de estudios es eficaz. } \\
\text { 50. }\end{array} \\
\begin{array}{l}\text { La transmisión de la información a los profesores es buena por } \\
\text { parte del equipo directivo. }\end{array}\end{array}$ \\
\hline & $\begin{array}{l}\text { Fuente de moti- } \\
\text { vación para los } \\
\text { profesores }\end{array}$ & $\begin{array}{l}\text { 53. Las decisiones relevantes, que competen al equipo docente, se } \\
\text { toman deliberando en común entre el equipo directivo y los } \\
\text { profesores. }\end{array}$ \\
\hline
\end{tabular}

Tabla 1. Dimensiones, indicadores e ítems finales del clima 


\section{Análisis de datos}

Tras un estudio inicial descriptivo de las respuestas, donde no se encontró ningún comportamiento irregular en la variabilidad y tendencia central de los ítems (medias entre 2,84 y 4,41, correspondientes a los ítems 10 y 35 , respectivamente -con desviaciones típicas de 1,02 y 0,87 - y desviaciones típicas que oscilan entre 0,67 y 1,17), se estudió la fiabilidad del instrumento calculando el alfa de Cronbach con el paquete informático SPSS 19, tanto para el cuestionario total como para cada una de las dimensiones que lo configuran, y analizando los índices de homogeneidad (correlación elemento-total corregida) de los ítems con el fin de determinar la conveniencia de la posible supresión de alguno de ellos (con valores inferiores a 0,2 según Hair et al., 2009, se suprime el ítem).

Posteriormente, con la ayuda de la herramienta informática AMOS 7.0, se determinó la bondad de ajuste del modelo factorial propuesto a nivel teórico, a través de las dimensiones e indicadores anteriormente expuestos, mediante un Análisis Factorial Confirmatorio siguiendo los criterios marcados por Byrne (2010) y Kline (2010) (CMIN/DF entre 2 y 5, CFI e IFI > 0,9, PRATIO, PNFI y PCFI > 0,9, RMSEA < 0,06 y HOELTER > 200) (ver tabla 2).

\section{RESULTADOS}

\section{Fiabilidad}

$\mathrm{Al}$ interpretar el alfa global correspondiente al instrumento inicial de medida de Clima de Centro se obtuvo un nivel de 0,947 ( $\alpha$ de Cronbach), no encontrando valores fuera de lo esperado en los índices de homogeneidad (por debajo de 0,2 según Hair et al., 2009). Cabe señalar que dichos valores no se vieron alterados tras las modificaciones realizadas al instrumento final, de acuerdo a los resultados del Análisis Factorial Confirmatorio. Por otro lado, se analizó también la fiabilidad del instrumento final por dimensiones, obteniendo niveles buenos ( $\alpha=0,842$ en la dimensión Relación Profesor-Alumno) y excelentes ( $\alpha=0,923$ y 0,964 en las dimensiones Relación Profesor-Compañeros y Relación Profesor-Superiores, respectivamente). Los valores del coeficiente de discriminación de los ítems finales oscilaron entre 0,29 y 0,78.

\section{Validez de Constructo (Análisis Factorial Confirmatorio)}

Habiéndonos fundamentado en la literatura consultada para la configuración de la estructura del instrumento (tabla 1), se realizó un análisis factorial confirmatorio mediante la aplicación de metodología S. E. M. (Structural Equation Modeling) para valorar la validez de constructo del instrumento. 
Para ello, se especificaron las reglas de correspondencia y relaciones entre las variables latentes y observadas que midió el cuestionario. De este modo, se propuso el Modelo inicial de medida (Figura 1) en el que se incluyeron todos los indicadores previstos en la teoría a fin de medir los tres constructos. Dicho modelo consta de 3 variables latentes, 60 variables observadas (de V01 a V60) y 60 términos de error (de e01 a e60). Asimismo, se definieron 60 cargas factoriales y 60 pesos de regresión entre los términos de error y sus variables asociadas. Se incluyeron las tres correlaciones entre los factores latentes principales y se consideraron incorrelacionados todos los términos de error.

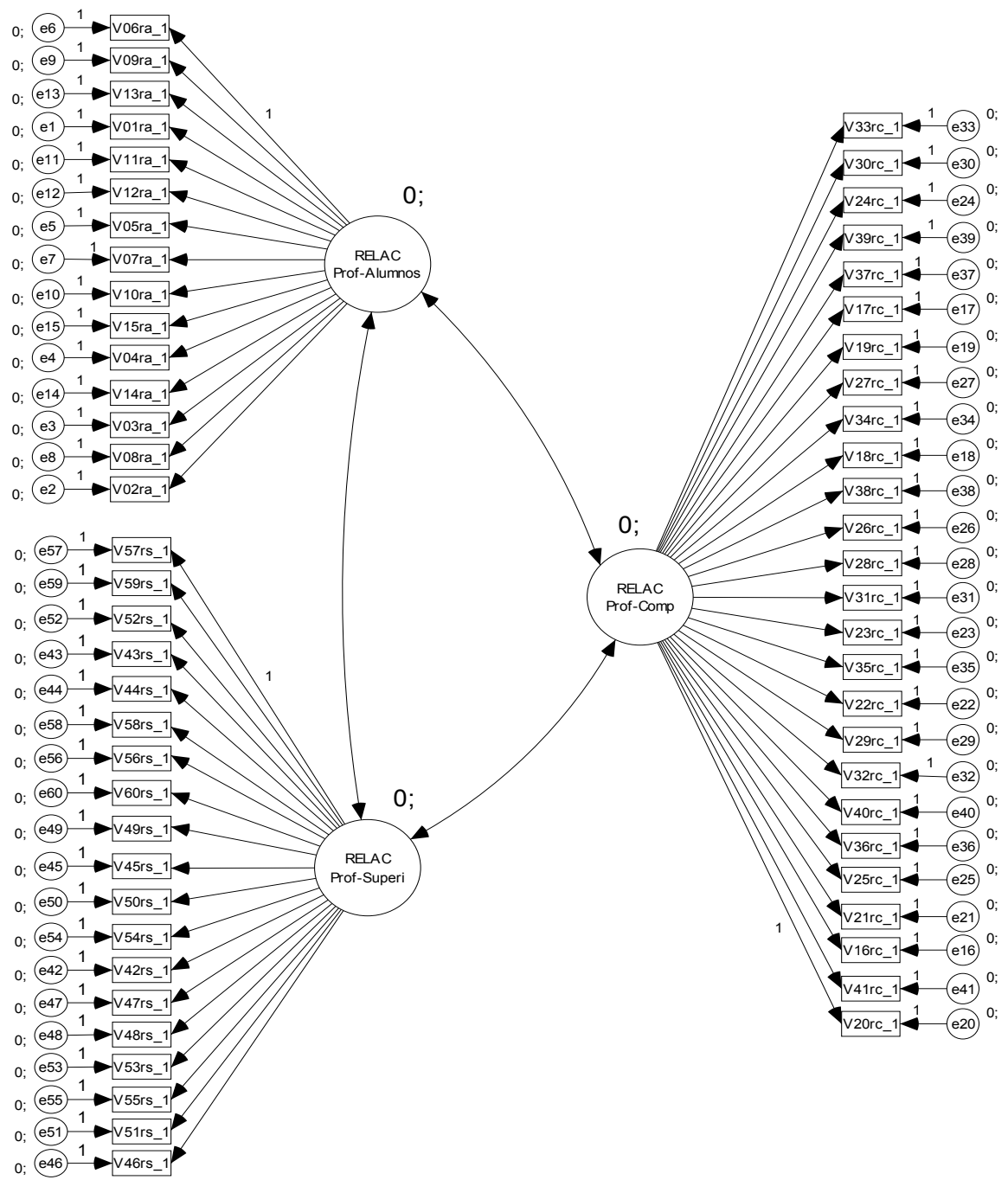

Figura 1. Modelo estructural inicial de medida del clima 
Una vez especificado el modelo, y asumida la normalidad multivariada (coeficiente de Mardia $=512,65$ menor que $p \cdot(p+2)$, siendo $p$ el número de variables observadas, 60 $(60+2)=3.720)($ Bollen, 1989), se procedió a la estimación de los parámetros del modelo por el procedimiento de Máxima Verosimilitud (Maximum Likelihood, «ML») -el más eficiente y no sesgado cuando se cumplen dichos supuestos de normalidad multivariante y lo suficientemente robusto como para no verse afectado por ligeras oscilaciones respecto de la distribución normal multivariada (Hayduk, 1996)-. Entre los resultados de este modelo (Tabla 2), encontramos cómo los índices de ajuste del modelo $\mathrm{CFI}=0,792$ y de IFI = 0,793 se sitúan por debajo del 0,90 requerido, según Kline (2010), debido, en parte, a cargas factoriales insatisfactorias de los ítems 2, 7, 12, 17, 24, 30 y 33 (por debajo del valor 0,5 señalado por Byrne, 2010, como necesario), por lo que dichos ítems fueron eliminados.

\begin{tabular}{|c|c|c|c|}
\hline Medida & $\begin{array}{l}\text { Nivel de ajuste } \\
\text { recomendado }\end{array}$ & $\begin{array}{c}\text { Valor } \\
\text { Modelo } \\
\text { Inicial }\end{array}$ & $\begin{array}{c}\text { Valor } \\
\text { Modelo } \\
\text { Final }\end{array}$ \\
\hline CMIN/DF & $2-5$ & 4,196 & 2,899 \\
\hline IFI & \multirow{2}{*}{$>0,9$} & 0,793 & 0,904 \\
\hline CFI & & 0,792 & 0,904 \\
\hline PRATIO & \multirow{3}{*}{$>0,7$} & 0,964 & 0,946 \\
\hline PNFI & & 0,718 & 0,814 \\
\hline PCFI & & 0,764 & 0,855 \\
\hline RMSEA & \multirow{3}{*}{$<0,06$} & 0,063 & 0,049 \\
\hline LO 90 & & 0,062 & 0,047 \\
\hline HI 90 & & 0,065 & 0,051 \\
\hline HOELTER.05 & \multirow{2}{*}{$>200$} & 200 & 293 \\
\hline HOELTER.01 & & 205 & 302 \\
\hline
\end{tabular}

Tabla 2. Resumen de los índices de ajuste de los modelos inicial y final de medida del Clima

Por otro lado, consultando la tabla de índices de modificación se encontraron correlaciones entre los términos de error de diversas variables (Tabla 3). Según esto, y basándonos en nuestra fundamentación teórica, parecía oportuna la inclusión de tres variables latentes: los factores Interacción con alumnos (en la dimensión Relación Profesor-Alumno), Conflictos entre compañeros (en la dimensión Relación Profesor-Compañeros) y Valoración de los superiores (en la dimensión Relación Profesor-Superiores) con el fin de comprobar si se producía una mejora en el ajuste del modelo. 


\begin{tabular}{|ccccc|}
\hline & & & M.I. & Par Chang. \\
\hline e15 & $<-->$ & e5 & 33,855 & 0,145 \\
e15 & $<-->$ & e14 & 70,877 & 0,163 \\
e10 & $<-->$ & e5 & 57,039 & 0,201 \\
e10 & $<-->$ & e14 & 59,783 & 0,160 \\
e10 & $<-->$ & e4 & 25,168 & 0,092 \\
e10 & $<-->$ & e15 & 130,14 & 0,314 \\
\hline e31 & $<-->$ & e28 & 72,069 & 0,240 \\
e23 & $<-->$ & e28 & 56,776 & 0,227 \\
e23 & $<-->$ & e31 & 66,15 & 0,190 \\
e35 & $<-->$ & e31 & 104,432 & 0,226 \\
e35 & $<-->$ & e28 & 52,546 & 0,206 \\
\hline e47 & $<-->$ & e48 & 25,946 & 0,071 \\
e48 & $<-->$ & e50 & 44,045 & 0,107 \\
e54 & $<-->$ & e48 & 85,766 & 0,143 \\
e42 & $<-->$ & e47 & 91,731 & 0,11 \\
\hline
\end{tabular}

Tabla 3. Índices de modificación I

Una vez incluidos los nuevos factores en el modelo, los índices de modificación (Tabla 4) desvelaron la conveniencia de algunas covariaciones entre términos de error que reducirían ostensiblemente el estadístico chicuadrado, siendo algunas de ellas justificables desde un punto de vista teórico. Concretamente, fueron factibles las correlaciones entre los términos de error e10 y e15 (ya que los dos aluden a cómo los alumnos acuden al profesor ante distintas problemáticas), entre e20 y e25 y entre e20 y e21 (pues hacen referencia a la implicación de los profesores en las actividades del centro), entre e 40 y e 32 (dado que se refieren a actitudes positivas en y hacia el centro) y entre e 38 y e18 (al hacer referencia a la percepción de la valoración que tienen los compañeros de uno mismo), entre e59 y e52 (ya que sus indicadores están referidos al liderazgo de los superiores), entre e53 y e55 (los elementos hacen referencia a la participación de los profesores en la toma de decisiones) y entre e58 y e60 (sus indicadores valoran aspectos derivados de la actitud y eficacia del director). De igual modo, se encontró una múltiple saturación de los ítems 45 (sobre la dimensión Relación Profesor-Compañeros y sobre la subdimensión Conflictos entre compañeros) y 54 (sobre las dimensiones Relación Profesor-Alumno y Relación ProfesoresCompañeros). Debido a esta multidimensionalidad, se optó por la eliminación de dichos ítems (Tabla 4). 


\begin{tabular}{|ccccc|}
\hline & & & M.I. & Par Change \\
\hline e10 & $<-->$ & e15 & 36,748 & 0,137 \\
e21 & $<-->$ & e20 & 61,805 & 0,155 \\
e25 & $<-->$ & e20 & 55,721 & 0,141 \\
e38 & $<-->$ & e18 & 96,111 & 0,114 \\
e40 & $<-->$ & e32 & 61,774 & 0,137 \\
e53 & $<-->$ & e55 & 74,353 & 0,140 \\
e59 & $<-->$ & e52 & 126,253 & 0,176 \\
e60 & $<-->$ & e58 & 50,277 & 0,096 \\
V54 & $<---$ & RPA & 12,76 & $-0,150$ \\
V54 & $<---$ & Conflic._Comp & 8,686 & $-0,119$ \\
V54 & $<---$ & RPC & 14,75 & $-0,148$ \\
V45 & $<---$ & RPC & 9,796 & $-0,138$ \\
V45 & $<---$ & Conflic._Comp & 4,73 & $-0,101$ \\
\hline
\end{tabular}

Tabla 4. Índices de modificación II

De este modo, y tras la realización de todas las modificaciones señaladas, se obtuvo un modelo final recursivo (Figura 2) estimado sobre una muestra de 794 sujetos, con 107 variables: 49 variables observadas (correspondientes a los ítems) y 58 variables latentes (6 son factores, 49 son términos de error y 3 términos de perturbación). De esas 107 variables, 55 son exógenas (49 términos de error, 3 términos de perturbación y 3 factores), y 52 son endógenas (49 indicadores y 3 factores). Por otro lado, son 161 parámetros a ser estimados, por lo que el modelo consta de 1.113 grados de libertad, resultando un modelo sobreidentificado y con posibilidad de ser estimado.

Los parámetros de dicho modelo final se estimaron, igualmente, por el procedimiento de Máxima Verosimilitud (coeficiente de Mardia = 390,07 menor que $49 \cdot(49+2)=2.499)$ y se alcanzaron resultados de estimación satisfactorios (Tabla 2), destacando un CFI $=0,904$, un IFI = 0,904; un CMIN/ DF de 2,899. Respecto a los residuos, alcanzamos un RMSEA de 0,049 y un tamaño muestral adecuado, pues el índice de Hoelter es de 302. Asimismo, los índices de parsimonia son elevados (PRATIO $=0,946$, PNFI $=0,814 \mathrm{y}$ PCFI $=0,855$ ), por lo que podemos afirmar que, teniendo en cuenta los parámetros que incorpora el modelo, nos encontramos ante un modelo de medida bastante parsimonioso.

En los índices de modificación no se encontraron valores dignos de tomar en consideración y analizando los valores estandarizados de los parámetros se constata la buena calidad de los indicadores, ya que las cargas factoriales son superiores a 0,5. Las correlaciones entre términos de error 
toman un valor apreciable en todos los casos (la menor es 0,25 ), así como las estimaciones de los términos de error (tabla que no se adjunta debido al tamaño de la misma).

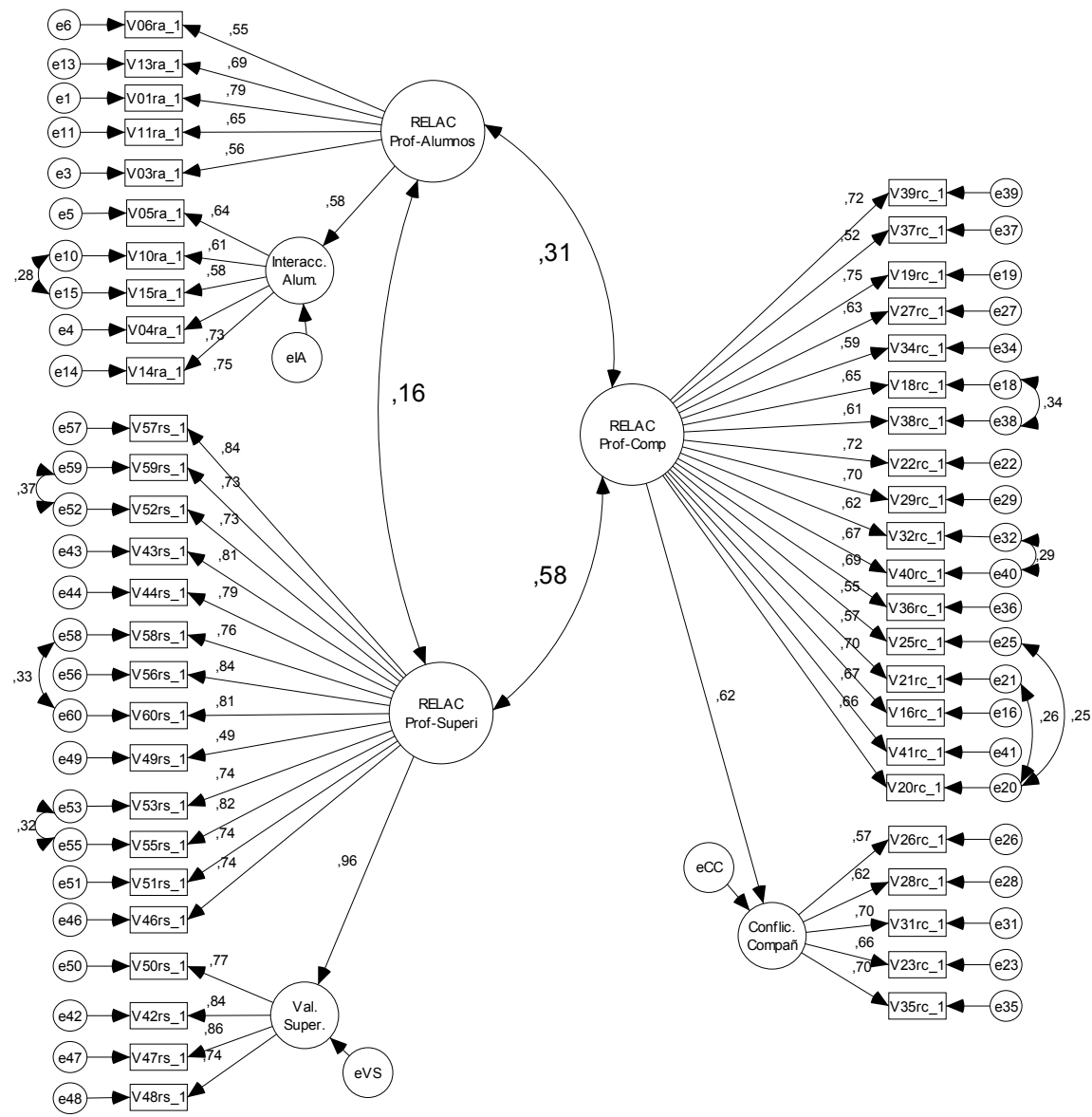

Figura 2. Modelo estructural final de medida del clima

Por último, y tomando como referencia los criterios de Hair et al. (2009), se observa que la dimensión Relación Profesor-Alumnos no muestra correlaciones significativas con el resto de dimensiones $(r$ Relación ProfesorAlumno-Relación Profesor-Compañeros: 0,31, $r$ Relación Profesor-Alumno-Relación Profesor-Superiores : 0,16), mientras que la Relación Profesor-Compañeros presenta una correlación moderada con Relación Profesor-Superiores ( $r$ Relación Profesor-Compañeros - Relación ProfesorSuperiores: 0,58). 


\section{DISCUSIÓN Y CONCLUSIONES}

Los resultados obtenidos han permitido presentar un instrumento para evaluar el clima en las organizaciones educativas, específicamente en centros ESO (ver Anexo), fundamentado teóricamente y definido operativamente, considerando tres dimensiones relevantes: Relación ProfesorAlumnos, Relación Profesor-Compañeros y Relación Profesor-Superiores. Esta aproximación no suele ser común en otros instrumentos de evaluación de clima de organizaciones, cultura, etc., por lo que ya de por sí constituye una aproximación relevante y diferenciada, tanto en su planteamiento teórico como en su operativización en ítems, consistente con el mismo. También el nivel educativo específico al que va dirigido representa no ya un instrumento más sino que aporta una orientación específica y diferente.

La elaboración rigurosa de su planteamiento teórico, bien fundamentado, ha determinado, en gran parte, que en el análisis de sus características técnicas la fiabilidad global sea excelente y exista una adecuada consistencia interna. Las escalas relativas a las relaciones mencionadas (Profesor-Alumnos, Profesor Compañeros y Profesor-Superiores) muestran niveles altamente satisfactorios, si bien se encuentra que la relación covariante entre la Relación Profesor-Alumnos es baja con el resto de las dimensiones, lo cual pone de manifiesto que la relación entre profesor y alumnos resulta bastante independiente respecto al resto de las relaciones. Este hecho, desde el punto de vista teórico, no resulta incoherente, ya que el microclima de aula no tiene por qué verse afectado o interferido por el resto de las interacciones que mantiene el profesor con otros agentes o grupos del centro -aspecto muy importante a tener en cuenta en otros estudios para profundizar en este punto y valorar en su justa medida la importancia de este tipo de relaciones en la consecución de los objetivos del centro-. Además, cabe señalar que el instrumento muestra una muy buena validez de contenido, avalada por la consistencia y rigor en la elaboración teórica y en las valoraciones que la selección de expertos en investigación educativa y de profesionales del ámbito de la ESO otorgaron respecto a la configuración de los ítems del instrumento.

Por otro lado, la validez de constructo quedó claramente probada en el análisis factorial confirmatorio con resultados muy satisfactorios y, en consecuencia, la adecuada inclusión de los ítems que componen el cuestionario, mostrando la coherencia entre la teoría y los ítems que se derivaron de la operativización de la misma. En este sentido, sin duda, uno de los elementos clave que ha posibilitado la realización de dicho análisis ha sido el tamaño muestral obtenido (Hair et al., 2009). No obstante, al encontrar una respuesta de participación moderada por parte de los profesores, consideramos pertinente ampliar el estudio a una muestra mayor con el fin de 
aumentar el poder de generalización del mismo. En esta línea, se debe tener en cuenta que el tipo de muestreo de carácter incidental, procedimiento común y justificado en este tipo de estudios por la voluntariedad del participante, puede afectar a la validez externa de la investigación, limitando el poder de generalización de los resultados. Del mismo modo, encontramos un cuarto tipo de relación interpersonal que consideramos importante para tener en cuenta en posteriores estudios: la relación entre profesor y familias (Murillo y Becerra, 2009) ya que, por ejemplo, en muchas ocasiones, el comportamiento disruptivo de los alumnos viene acompañado por una falta de apoyo de las familias hacia el profesorado.

Por otro lado, los estudios realizados a través del mencionado análisis factorial confirmatorio mostraron, igualmente, una sólida y robusta configuración de las dimensiones y subdimensiones que subyacen al instrumento: la Relación Profesor-Alumno (a través del comportamiento de los alumnos, la interacción del profesor con los estudiantes y la motivación de los mismos), de la Relación Profesor-Compañeros (compuesto por el trabajo en equipo, el nivel de profesionalidad de los compañeros, el nivel de conflictos entre profesores y la interacción entre ellos) y de la Relación Profesor-Superiores (a través del liderazgo de los directores y jefes de estudio, la valoración profesional de los mismos y su papel motivador para los profesores) al encontrar saturaciones adecuadas de los ítems sobre los factores del clima. En este sentido, reiteramos la importancia de la fundamentación teórica y la propia consistencia del proceso, determinante de los resultados y conclusiones derivadas de los mismos.

En síntesis, el instrumento propuesto constituye una aportación relevante, tanto desde el punto de vista teórico, por la aproximación en la elaboración (basada en las mencionadas dimensiones) y por el nivel educativo específico de ESO al que va dirigido, así como por sus características técnicas relativas a su fiabilidad y validez. En este sentido, se ha mostrado la consistencia del instrumento a través de la validez de constructo y la satisfactoria estructura dimensional propuesta, por lo que, a modo de conclusión y de acuerdo al objetivo de este trabajo, se puede afirmar que se ha contribuido al campo científico del estudio del clima de los centros con un instrumento de medida diferente, válido y fiable. La importancia de este constructo en el logro de los objetivos de los centros educativos hace que se precisen más y mejores investigaciones en este campo, a través de instrumentos que permitan medidas adecuadas de los factores considerados. 


\section{REFERENCIAS BIBLIOGRÁFICAS}

Aciego, R., Domínguez, R. y Hernández, P. (2003). Evaluación de la mejora en valores de realización personal y social en adolescentes que han participado en un programa de intervención. Psicothema, 15(4), 589-594.

Arciniega, L. (2002). Compromiso organizacional México ¿Cómo hacer que la gente se ponga la camiseta? Dirección estratégica, 11, 21-23.

Baeza, S. (2005). Funcionamiento y clima sociorrelacional del aula. Una perspectiva sistémica: una escala sistémica de observación de clases. Buenos Aires: Editorial Aprendizaje Hoy.

Byrne, B. (2010). Structural equation modelling with AMOS. (2nd ed.). New York: Taylor and Francis Group.

Cano-García, F. J., Padilla-Munoz, E. M. y Carrasco-Ortiz, M. A. (2005). Personality and contextual variables in teacher burnout. Personality and Individual Differences, 38, 929-940.

Collell, J. y Escudé, C. (2006). Maltrato entre alumnos (I). Presentación de un cuestionario para evaluar les relaciones entre iguales. CESC Conducta y experiencias sociales a clase. Ámbitos de Psicopedagogía, 12, 812-825.

Consejería de Educación de Madrid (2013). Bases de datos del Instituto de Estadística de la C. A. M. Madrid: Consejería de Educación, Comunidad Autónoma de Madrid.

Cortada de Kohan, N. (1999). Teorías psicométricas y Construcción de Tests. Buenos Aires: Editorial Lugar.

Esparza, C., Guerra, P. y Martínez, M. (2000). Determinación de los niveles del síndrome burnout en profesionales de la salud de la V región. Revista de Psicología Universidad de Valparaíso, 1, 73-95.
Esteve, J. M. (2009). La profesión docente en Europa: perfil, tendencias y problemática. La formación inicial. Revista de Educación, 340, 19-86.

Fernández Díaz, M. J. y Asensio Muñoz, I. (1993). Evaluación del clima de centros educativos. Revista de Ciencias de la Educación, 153, 69-83.

Gillespie, N. A., y Mann, L. (2004). Transformational leadership and shared values: the building blocks of trust. Journal of Managerial Psychology, 19(6), 588-607.

Guerra, C., Vargas, J., Castro, L., Plaza, H. y Barrera, P. (2012). Percepción del Clima Escolar en estudiantes de Enseñanza Media de Valparaíso de colegios municipales, subvencionados y particulares. Estudios Pedagógicos, 38, 103-115.

Hair, J., Anderson, R., Tathan, R. y Black, W. (2009). Análisis multivariante. Madrid: Pearson.

Hayduk, L. A. (1996). LISREL Issues, Debates and Strategies. Baltimore, USA: Johns Hopkins University Press.

Haynes, N. M., Emmons, C. L. y Ben-avie, M. (1997). School climate as a factor in student adjustment and achievement. Journal of Educational and Psychological Consultation, 8, 321-329.

Hernández, G. y Olmedo, E. (2004). Un estudio correlacional acerca del síndrome del "estar quemado» (burnout) y su relación con la personalidad. Andalucía occidental, 22(1), 121-136.

Hoy, W. K. y Feldman, J. A. (1999). Organizational Health Profiles for Higher Schools. London: Ed. H. J. Freiberg.

Informe Cisneros XI (2009). Libro Blanco. Los riesgos psicosociales en el 
profesorado de la enseñanza pública en la Comunidad de Madrid. Sindicato ANPE. Madrid: ANPE.

Jennings, P. A. y Greenberg, M. T. (2009). The Prosocial Classroom: Teacher Social and Emotional Competence in Relation to Student and Classroom Outcomes. Review of Educational Research, 79(1), 491-525.

Kelley, E. A. (1989). Improving school climate. Practitioner, 15(4), 1-5.

Kline, R. (2010). Principles and practice of structural equation modelling (Third edition). New York: The Guilford Press.

Kuperminc, G. P., Leadbeater, B. J. y Blatt, S. J (2001). School social climate and individual differences in vulnerability to psychopathology among middle school students. Journal of School Psychology, 39, 141-159.

Lozano, L. (2013). Impacto de los estilos de liderazgo en el clima institucional del Bachillerato de la Unidad Educativa María Auxiliadora de Riobamba. Alteridad. Revista de Educación, 2(8), 192-206.

Marzano, R. J., Marzano, J. S. y Pickering, D. J. (2003). Classroom management that works. Alexandria, VA: ASCD.

Mejías A., Reyes, S. y Arzola, M. (2006). Medición del clima organizacional en instituciones de educación superior. Universidad, Ciencia y Tecnoclogía, 10, 55-61.

Molina, N. $y$ Pérez, I. (2006). El clima de relaciones interpersonales en el aula un caso de estudio. Paradigma, 27, 193-219.

Murillo, P. y Becerra, S. (2009). Las percepciones del clima escolar por directivos, docentes y alumnado mediante el empleo de «redes semánticas naturales». Su importancia en la gestión de los centros educativos. Revista de Educación, 350, 375-399.

Pérez, A., Ramos, G. y López, E. (2009). Diseño y análisis de una escala para la valoración de la variable clima social aula en alumnos de Educación Primaria y Secundaria. Revista de Educación, 350, 221-252

Rivas, J. I., Leite, A. E. y Cortés, P. (2011). Paradojas y conflictos entre las culturas del profesorado, las familias y los estudiantes en el contexto escolar. Revista de Educación, 356, 161-183.

Rodríguez Mantilla, J. M. y Fernández Díaz, M. J. (2012). El síndrome de Burnout en el profesorado de Secundaria y su relación con variables personales y profesionales. Revista Española de Pedagogía, 252(70), 259-278.

Rodríguez M., D. (2005). Diagnóstico Organizacional (6ta edición). México: Ediciones Universidad Católica de Chile.

Romasz, T. E., Cantor, J. H. y Elias, M. J. (2004). Implementation and evaluation of urban school-wide social-emocional learning programs. Evaluation and Program Planning, 27, 89-103.

Rosenblatt, J. A. $y$ Furlong, M. J. (1997). Assessing the Reliability and Validity of Student Self-Reports of Campus Violence. Journal of Youth and Adolescence, 26(2), 187-202.

Ruiz-Primo, A., Jornet, J. M. y Backhoff, E. (2006). Acerca de la validez. de los exámenes de calidad y logro educativos (EXCALE). México: Instituto Nacional para la Evaluación de la Educación.

Teixidó Saballs, J. y Capell Castañer, D. (2002). Formación del profesorado orientada al desarrollo de competencias de gestión del aula de ESO; el afrontamiento de situaciones críticas. 
Revista Electrónica Interuniversitaria de Formación del Profesorado, 5, 110-134.

Tejero, C. (2006). Burnout y dirección escolar: análisis de la influencia que sobre el sindrome ejercen las variables perfil demográfico-profesional, estrés, satisfacción e indefensión. Universidad Complutense de Madrid. (Tesis Doctoral).

Tejero, C., Fernández, M. J. y Carbajo, R. (2010). Medición y prevalencia del síndrome de quemarse por el trabajo (burnout) en la dirección escolar. $R e$ vista de Educación, 351, 361-383.
Trianes, M. V., Blanca, M. J., De la Morena, L., Infante, L. y Raya, S. (2006). Un cuestionario para evaluar el clima social del centro escolar. Pshicothema, 18(2). 272-277.

Westling, M. (2002). A two level analysis of classroom climate in relation to social context, group composition and organization of special support. Learning Environments Research, 5, 253-274.

Wilkins, N. y Kuperminc, G. P. (2010). Why try? Achievement motivation, perceived academic climate and academic outcomes among Latino youth. Journal of Early Adolescence, 30, 246-276. 


\section{ANEXO}

\section{INSTRUMENTO DE MEDIDA DEL CLIMA DE CENTRO}

Por favor, indique los datos que más se ajustan a su persona y centro de trabajo marcando con una «X» en los recuadros que corresponda.

$\begin{array}{rlll}1 \text { SEXO: } & \square \text { Hombre } \quad \square \text { Mujer } \\ & \text { ESTADO CIVIL: } & \square \text { Soltero/a } \\ & \square \text { Casado/a } \\ & \square \text { Separado/a - Divorciado/a } \\ & \square \text { Religioso/a } \\ & \square \text { Viudo/a }\end{array}$

3 NÚMERO DE HIJOS/AS: $\square$ Ninguno $\square 1$ o $2 \square 3$ o más

4 EDAD: $\square 29$ o menos $\square$ 30-39 $\square$ 40-49 $\square 50$ o más

5 AÑOS DE ANTIGÜEDAD COMO PROFESOR A LO LARGO DE TODA SU CARRERA:

6 TITULARIDAD DEL CENTRO: $\square$ Público $\square$ Privado $\square$ Privado Concertado

7 CICLO DE E. S. O. EN EL QUE IMPARTE CLASES: $\square 1^{\text {er }}$ Ciclo

$\square 2 .^{\circ}$ Ciclo

$\square$ Ambos ciclos

8 ASIGNATURA/S QUE IMPARTE ACTUALMENTE:

$\square$ Ciencias (Matemáticas, Ciencias Naturales, Física, Química. Biología, Geología, Botánica)

$\square$ Humanidades (Lengua, Literatura, Ciencias Sociales, Geografía, Historia, Cultura Clásica)

$\square \quad$ Lengua extranjera (Inglés, Francés, Alemán, Latín, Griego etc.)

$\square$ Artes (Música, Tecnología, Educación Plástica y Visual)

$\square$ Religión

$\square \quad$ Educación Física

$\square$ Otra:

9 CARÁCTER DE LA/S ASIGNATURA/S QUE IMPARTE

$\square$ Obligatoria $\quad \square$ Optativa

10 PUESTO DE TRABAJO DESEMPEÑADO

$\square$ Profesor y Tutor

$\square$ Solo Profesor de Área

$\square$ Profesor y miembro de Equipo Directivo

11 TITULACIÓN MÁXIMA QUE POSEE: $\square$ Diplomado/a o ingeniero técnico

$\square$ Licenciado/a o ingeniero superior

$\square$ Doctor/a

A continuación le presentamos una serie de enunciados con el objetivo valorar el clima de centro. Para ello, valore cada unos de los siguientes ítems que hacen referencia a su relación con los alumnos, compañeros y superiores del centro, en una escala de 1 a 5 , siendo 1 el valor mínimo (que indica NADA, NUNCA) y 5 el valor máximo (que indica MUCHO, SIEMPRE). 


\begin{tabular}{|c|c|c|c|c|}
\hline & $\begin{array}{l}\text { Nada } \\
\text { Nunca }\end{array}$ & & & \\
\hline $\begin{array}{l}\text { 1. En general, en mis clases, los alumnos permiten que se im- } \\
\text { parta la clase }\end{array}$ & 12 & 3 & & \\
\hline 2. En mis clases los alumnos participan activamente & 12 & 3 & 4 & \\
\hline 3. Existe una buena comunicación entre mis alumnos y yo & 12 & 3 & $4 \quad 5$ & \\
\hline $\begin{array}{l}\text { 4. Los alumnos acuden a mí cuando tienen dificultades acadé- } \\
\text { micas y no solo por cuestiones de examen }\end{array}$ & 12 & & 45 & \\
\hline $\begin{array}{l}\text { 5. Durante mis clases los alumnos utilizan «palabrotas» o ma- } \\
\text { las formas en el aula }\end{array}$ & 12 & & 45 & \\
\hline 6. Los alumnos me confían sus temas o problemas personales & 12 & 3 & 4 & \\
\hline 7. Los alumnos respetan la autoridad de los profesores. & 12 & 3 & 45 & \\
\hline 8. En mi clase se respeta & 12 & 3 & $4 \quad 5$ & \\
\hline 9. Mi relación con los alumnos es cercana & 12 & 3 & 4 & \\
\hline 10. Cuando existen conflictos entre mis alumnos, acuden a mí & 12 & 3 & $4 \quad 5$ & \\
\hline $\begin{array}{l}\text { 11. Existe una implicación y ac } \\
\text { profesores a la hora de trab }\end{array}$ & 12 & & & \\
\hline 12. Me siento valorado por mis compaî & 12 & 3 & 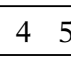 & \\
\hline $\begin{array}{l}\text { 13. A nive } \\
\text { nes en }\end{array}$ & 12 & $\mathrm{~J}$ & 45 & \\
\hline 14. El profesorado & 12 & & 1. & \\
\hline $\begin{array}{l}\text { 15. En gen } \\
\text { uno a } 1\end{array}$ & 12 & & 45 & \\
\hline $\begin{array}{l}\text { En gener } \\
\text { tro es bue }\end{array}$ & 12 & & 45 & \\
\hline $\begin{array}{l}\text { 17. He tenid } \\
\text { fesores/a }\end{array}$ & 12 & & & \\
\hline $\begin{array}{l}\text { 18. A la hora de preparar ciertos eventos (Navid } \\
\text { ciencia, etc.) los profesores colaboran. }\end{array}$ & 12 & & & \\
\hline $\begin{array}{l}\text { 19. Hay un ambi } \\
\text { el profesorad }\end{array}$ & 12 & & & \\
\hline 20. Mantengo buenas relaciones con todos mis compañeros. & 12 & & 7 & \\
\hline $\begin{array}{l}\text { 21. Siento que la actitud de algunos compañeros me dificulta } \\
\text { expresar mis opiniones. }\end{array}$ & 12 & & & \\
\hline $\begin{array}{l}\text { 22. En general, los profesores prestan su ayuda a un compañero } \\
\text { cuando tiene algún problema o dificultad }\end{array}$ & 12 & & & \\
\hline Me siento criticado negativamente por otros profesores & 12 & 3 & 4 & \\
\hline 24. El profesorado manifiesta optimismo, energía y entusiasmo & 12 & & 4 & \\
\hline $\begin{array}{l}\text { 25. Las propuestas que hago son tenidas en cuenta por mis } \\
\text { compañeros }\end{array}$ & & & & \\
\hline
\end{tabular}




\begin{tabular}{|c|c|c|c|c|}
\hline & & $\begin{array}{l}\text { Nada } \\
\text { Nunca }\end{array}$ & $\begin{array}{r}\text { Mucl } \\
\text { Siemp }\end{array}$ & $\begin{array}{l}\text { cho } \\
\text { ipre }\end{array}$ \\
\hline & $\begin{array}{l}\text { He tenido o tengo conflictos de tipo profesional con otros } \\
\text { profesores }\end{array}$ & 12 & 34 & 5 \\
\hline 27. & $\begin{array}{l}\text { Me siento satisfecho con el trabajo que desarrollan los pro- } \\
\text { fesores en general. }\end{array}$ & 12 & 34 & 5 \\
\hline & $\begin{array}{l}\text { En general, los profesores cumplen con los horarios de vigi- } \\
\text { lancias (por ejemplo: guardias de recreo, vigilancia de pasi- } \\
\text { llos, etc.) }\end{array}$ & 12 & 34 & 5 \\
\hline 29. & $\begin{array}{l}\text { En general, creo que la opinión que tienen mis compañeros } \\
\text { de mí es buena }\end{array}$ & 12 & 34 & 5 \\
\hline & $\begin{array}{l}\text { Considero que el profesorado actúa en su trabajo con profe- } \\
\text { sionalidad }\end{array}$ & 12 & 34 & 5 \\
\hline & $\begin{array}{l}\text { Considero que el profesorado se siente orgulloso de su cen- } \\
\text { tro. }\end{array}$ & 12 & 4 & 5 \\
\hline & $\begin{array}{l}\text { En general, los profesores comparten materiales y recursos } \\
\text { con otros profesores }\end{array}$ & 12 & 34 & 5 \\
\hline & $\begin{array}{l}\text { La jefatura de estudios atiende las necesidades (materiales, } \\
\text { de información, etc.) de los profesores }\end{array}$ & 12 & 34 & 5 \\
\hline & $\begin{array}{l}\text { Ante un problema profesional de algún profesor, la jefatura } \\
\text { de estudios (o dirección) responde de forma eficaz }\end{array}$ & 12 & 34 & 5 \\
\hline & $\begin{array}{l}\text { El Equipo Directivo se muestra flexible a la hora de tener } \\
\text { que modificar sus decisiones. }\end{array}$ & 12 & 3 & 5 \\
\hline & $\begin{array}{l}\text { El Equipo Directivo pone los medios para potenciar la ac- } \\
\text { tualización profesional de los docentes. }\end{array}$ & 12 & 34 & 5 \\
\hline 37. & Considero que la jefatura de estudios hace un buen trabajo & 12 & 34 & \\
\hline & $\begin{array}{l}\text { La gestión y organización de eventos, tareas, etc. por parte } \\
\text { del equipo directivo es eficaz }\end{array}$ & 12 & 34 & 5 \\
\hline & $\begin{array}{l}\text { La línea de actuación del equipo directivo se fundamenta } \\
\text { más en la improvisación que en la previsión o claridad de } \\
\text { ideas }\end{array}$ & 12 & 3 & 5 \\
\hline 40. & $\begin{array}{l}\text { La transmisión de la información a los profesores es buena } \\
\text { por parte del equipo directivo }\end{array}$ & 12 & 34 & 5 \\
\hline & $\begin{array}{l}\text { En general, el equipo directivo se esfuerza por reconocer el } \\
\text { trabajo de los profesores (reconocimientos, recompensas, } \\
\text { etc.) }\end{array}$ & 12 & 34 & 5 \\
\hline & $\begin{array}{l}\text { El jefe de estudios mantiene un trato adecuado con los pro- } \\
\text { fesores }\end{array}$ & 12 & 34 & \\
\hline & $\begin{array}{l}\text { Las decisiones relevantes, que competen al equipo docente, } \\
\text { se toman deliberando en común entre el equipo directivo y } \\
\text { los profesores }\end{array}$ & 12 & 34 & 5 \\
\hline 44. & $\begin{array}{l}\text { El Equipo Directivo respeta las decisiones tomadas demo- } \\
\text { cráticamente y acepta las sugerencias que se le hacen. }\end{array}$ & 12 & 34 & 5 \\
\hline
\end{tabular}




\begin{tabular}{|l|llllll|}
\hline & & $\begin{array}{l}\text { Nada } \\
\text { Nunca }\end{array}$ & $\begin{array}{c}\text { Mucho } \\
\text { Siempre }\end{array}$ \\
\hline 45. & $\begin{array}{l}\text { El Equipo Directivo mantiene buenas relaciones con el pro- } \\
\text { fesorado en general. }\end{array}$ & 1 & 2 & 3 & 4 & 5 \\
\hline 46. & $\begin{array}{l}\text { Existe una buena comunicación entre el profesorado y los } \\
\text { cargos de responsabilidad (jefe de estudios, dirección, etc. })\end{array}$ & 1 & 2 & 3 & 4 & 5 \\
\hline 47. & El director mantiene un trato adecuado conmigo & 1 & 2 & 3 & 4 & 5 \\
\hline 48. & $\begin{array}{l}\text { El jefe de estudios y yo mantenemos una buena comunica- } \\
\text { ción sobre cuestiones profesionales }\end{array}$ & 1 & 2 & 3 & 4 & 5 \\
\hline 49. & Los profesores confiamos profesionalmente en el director & 1 & 2 & 3 & 4 & 5 \\
\hline
\end{tabular}

Muchas gracias por su colaboración. 


\section{PERFIL ACADÉMICO Y PROFESIONAL DE LOS AUTORES}

Jesús Miguel Rodríguez Mantilla, Doctor en Educación, Licenciado en Psicopedagogía y Diplomado en Magisterio de Educación Primaria por la Universidad Complutense de Madrid, es profesor de Métodos de Investigación en la Universidad Camilo José Cela y tutor de dicha asignatura y de Diseños de Investigación en la UNED. Es también profesor de Organización del Centro Escolar, Programación Docente en el nuevo marco de las Competencias, Resolución de Conflictos y Toma de Decisiones en Másteres Oficiales.

M. José Fernández Díaz, Decana de la Facultad de Educación de la UCM y Profesora del Departamento de Métodos de Investigación y Diagnóstico en Educación. Sus principales áreas de especialización son: metodología de investigación; evaluación y medida en educación; dirección y liderazgo, formación de profesores y calidad y evaluación de centros. Es evaluadora de proyectos de investigación en distintas agencias, entre ellas la ANEP.

Dirección de los autores: Jesús Miguel Rodríguez Mantilla

Facultad de Educación

Universidad Camilo José Cela

C/ Castillo de Alarcón, 49

Urb. Villafranca del Castillo

28692 (Madrid)

E-mail: jmrodriguez@ucjc.edu

M. ${ }^{a}$ José Fernández Díaz
Facultad de Educación
Universidad Complutense de Madrid
C/ Rector Royo Villanova, s/n
28040 (Madrid)
E-mail: mjfidaz@edu.ucm.es

Fecha Recepción del Artículo: 15. Marzo. 2012

Fecha Modificación Artículo: 11. Diciembre. 2012

Fecha Aceptación del Artículo: 19. Diciembre. 2012

Fecha Revisión para publicación: 15. Mayo. 2014 
